\title{
Hormonal responses to graded-resistance, FES-assisted strength training in spinal cord-injured
}

\author{
GD Wheeler ${ }^{1}$, EA Ashley ${ }^{2}$, V Harber ${ }^{1}$, JJ Laskin ${ }^{1}$, LM Olenik ${ }^{1}$, D Sloley ${ }^{3}$, R Burnham ${ }^{1}$, RD Steadward ${ }^{1}$ and \\ DC Cumming ${ }^{4}$
}

${ }^{I}$ The Rick Hansen Centre, University of Alberta, Edmonton, Alberta; ${ }^{2}$ Institute of Physiology, University of Glasgow, Scotland; ${ }^{3}$ Department of Zoology, University of Alberta; ${ }^{4}$ Department of Obstetrics and Gynaecology and Medicine (Division of Endocrinology), University of Alberta, Canada

\begin{abstract}
Functional electrical stimulation (FES) assisted resistance training has been effective in increasing muscular strength and endurance in spinal cord injured men and women in preparation for FES-assisted cycle programs and for FES-assisted standing and walking. Increases in blood pressure and a concomitant bradycardia suggestive of autonomic dysreflexia have been reported during FES-assisted resistance training. Self-induced autonomic dysreflexia in athletes who use wheelchairs suppressed the normal exercise induced serum testosterone increase. We, therefore, examined the changes in hematocrit and circulating levels of testosterone, sex hormone binding globulin (SHBG), cortisol, prolactin, norepinephrine and epinephrine during FES assisted resistance exercise in five high spinal cord injured men (SCI) and comparable maximal exercise in five able bodied controls (AB). Mean serum testosterone levels significantly increased with FES-assisted resistance training in SCI and maximal resistance exercise in $\mathrm{AB}$ with no significant change in hematocrit or SHBG. Prolactin, cortisol and epinephrine levels were unchanged while norepinephrine levels were significantly increased in SCI and AB. These findings suggest that there is no concern over inadequate physiological androgen response to an exercise stimulus in SCI. The data do not support the previous findings that elevated levels of norepinephrine in autonomic dysreflexia suppress testosterone response to exercise.
\end{abstract}

Keywords: functional electrical stimulation; catecholamines; norepinephrine; epinephrine; testosterone; cortisol

\section{Introduction}

The endocrine system at rest is characterized by endogenous rhythmic fluctuations in hormone levels. Superimposed on the internal rhythms is the capacity to respond to changes in the external environment. Thus, in the short term, stress, feeding, trauma, physical activity and other stimuli are followed by adjustments in hormonal control. Chronic alterations in the environment may also induce changes in hormonal milieu. In the able-bodied, serum total and free testosterone levels increase during short term strenuous exercise and decrease during and after prolonged activity. ${ }^{1,2}$ While the mechanism(s) and effects of such changes remain(s) disputed, recent evidence suggests that testosterone is 'consumed' during and subsequent to prolonged, strenuous physical activity. ${ }^{3}$ It is tempting to believe that testosterone consumption during and following activ-

Correspondence: DC Cumming, 1D1 Walter Mackenzie Health Sciences Centre, University of Alberta, Edmonton, Alberta, Canada T6G 2R7 ity may be important in tissue repair in muscle and bone. ${ }^{4}$

Spinal cord injury (SCI) has been reported to cause both acute and long term changes in the endocrine milieu in at least some individuals. ${ }^{5-10}$ Physical activity induced hormonal changes have been little studied in individuals who use wheelchairs. ${ }^{11-13}$ Such responses could be important for several reasons; for example, abnormal responses to exercise and training may limit wheelchair users' tolerance of increased activity, or may limit muscle or bone development with training.

Functional electrical stimulation (FES) inducing muscular contract has been used to increase muscle mass, increase bone density, reduce decubitus ulcers, and to improve cardio-pulmonary fitness. ${ }^{14-16}$ Autonomic dysreflexia-like responses and exaggerated increases in norepinephrine may occur with FES. ${ }^{13,17}$ This may be a limiting factor on the ability to use FES in training individuals who use wheelchairs. We have also reported that 'boosting' ie the use of insensate painful stimuli to induce neurogenic hypertension 
inhibits the normal acute exercise induced testosterone response in elite paraplegic athletes. ${ }^{11}$ It is therefore appropriate to determine the hormonal responses to FES assisted strength training. The purpose of the investigation, therefore, was to examine hormonal responses to acute maximal FES-assisted resistance exercise in SCI and comparable maximal voluntary exercise in $\mathrm{AB}$ males.

\section{Subjects and methods}

The subjects in the study were five spinal cord-injured and five able-bodied men. All subjects gave informed consent to take part in the investigation which was approved by the institutional ethics committee. All SCI subjects were in good health; their participation was approved by the medical advisor at the Rick Hansen Centre, University of Alberta, where the investigations were performed. All SCI subjects had undergone bone densitometry using a dual photon Xray absorptiometry machine to eliminate the possibility of stress fractures and had routinely received FES at the Rick Hansen Centre.

On arrival at the laboratory the subject mounted or was transferred to a specially adapted hydraulic resistance training device ('Hydrastim'-Biomech Designs, Edmonton, Ltd). A cannula was inserted into a forearm vein and kept patent via continuous saline infusion. Blood samples were subsequently obtained through a system of three way stop cocks as previously described. ${ }^{18}$

The hydraulic resistance training device consists of a modified Hydragym hydraulic leg extension and flexion device (Hydragym Canada, Ltd) interfaced with an IBM computer (IBM. Model PS2 80). Modifications included electronic lever arm goniometers and force transducers mounted on extended lever arm leg pads. Leg pads were also enlarged to maximize force dissipation across the anterior portion of the tibia. The computer system delivered muscle stimulation via a computer controlled 4 channel neuromuscular stimulation device ('Hydrastim' Biomech Designs, Edmonton, Ltd.) connected to the subject via re-usable surface electrodes (Chattanooga Corp, TN, USA). Stimulation parameters included square monophasic waves, 250 millisecond pulse width,@25 Hz,@0-150mA and 50\% duty cycle (set at $4 \mathrm{~s}$ on/off time).

Subjects remained seated for a $1 \mathrm{~h}$ equilibration period after the insertion of the intravenous catheter. A resting blood sample $(10 \mathrm{ml})$ was taken after $1 \mathrm{~h}$ and repeated after a further $15 \mathrm{~min}$. Electrodes were then applied in SCI subjects to the quadriceps muscle $5 \mathrm{~cm}$ proximal to the superior margin of the patella and at a motor point of the rectus femoris/vasti group. The hydraulic cylinder was set at 1 and a maximal stimulus (160 mAMP ramped over a $15 \mathrm{~s}$ period) was applied to the limbs sufficient to achieve full and rapid extension of the leg ( $>170$ degrees) for a period of 5 min (approximately 25 contractions).
Able-bodied subjects worked voluntarily at a maximal level at setting 1 on the Hydragym for a period of 5 min sufficient to produce a minimum of 25 contractions per limb. Work rate was set by a metronome. This was repeated at settings 3 and 5 on the Hydrastim apparatus at voluntary (AB) or FESstimulated (SCI) maximal effort. A $10 \mathrm{ml}$ blood sample was taken at the end of each work bout followed by a $15 \mathrm{~min}$ rest period to facilitate full recovery of the muscle. This protocol was designed to produce maximal force output while allowing for recovery in both the $\mathrm{AB}$ and SCI groups. A final blood sample was taken at the end of the third workload and $15 \mathrm{~min}$ thereafter. No motivation was given to the $\mathrm{AB}$ group to minimize confounding of hormone responses by psychogenic arousal.

Each blood sample was aliquoted for analysis. A sample for hematocrit was taken immediately in a heparinized micropipet. Half of each remaining sample was treated for catecholamine assay of epinephrine (EPI) and norepinephrine (NEP) and stored at $-80^{\circ} \mathrm{C}$ as previously reported (Burnham et al 1993). The remainder was allowed to clot, was centrifuged and the serum separated and stored at $-20^{\circ} \mathrm{C}$ for later assay of total testosterone $(\mathrm{T})$, cortisol $(\mathrm{F})$, prolactin $(\mathrm{PRL})$ and sex hormone binding globulin (SHBG). Total T, $\mathrm{C}, \mathrm{PRL}$ and $\mathrm{SHBG}$ were assayed with commercially available RIA kits (Immunocorp Co-T/C/ PRL.IRMA Count, Diagnostic Products Corporation, LA, USA - SHBG).

An analysis of variance (ANOVA) with repeated measures was performed on the data. Post-hoc comparisons between time 1, 2 and 3 measures between groups were conducted using a Scheffé Test. All data were normalized prior to the analysis. Changes from baseline measures were calculated for each individual and ANOVA was computed for the residuals. An alpha level of $P<0.05$ was determined a priori for an acceptable level of statistical significance.

\section{Results}

As shown in Table 1, serum testosterone levels increased significantly throughout the exercise in both SCI $(P<0.01)$ and $\mathrm{AB} \quad(P<0.05)$ subjects without change in hematocrit or SHBG (both $P>>0.05$ ). Baseline total $\mathrm{T}$ levels were significantly higher in SCI versus $\mathrm{AB}$ and this difference was maintained throughout the exercise session $(P<0.0001)$. Both values were within the normal range for men. Serum testosterone levels in SCI rose from $24.1 \pm 3.5$ (SEM) to $30.1 \pm$ $5.2 \mathrm{nmol} / \mathrm{l}$ and in $\mathrm{AB}$ from $15.2 \pm 1.7$ to $19.1 \pm$ $1.9 \mathrm{nmol} / \mathrm{l}$. PRL levels were not significantly different between the groups and were unchanged with exercise. $F$ levels were not significantly different at rest and tended to fall during the course of the exercise in both groups although this was not significant.

As shown in Table 1, Norepinephrine levels were significantly increased with each exercise in SCI and AB $(P<0.01)$. NOR levels increased from $2.5 \pm 0.3$ to 
Table 1 Mean \pm SEM hormonal responses to graded-resistance, FES-assisted strength training in 5 spinal cord-injured (SCI) and 5 Able-bodied (AB) subjects

\begin{tabular}{|c|c|c|c|c|c|c|c|c|}
\hline Work period & & $\begin{array}{c}\text { Testosterone } \\
(\mathrm{nmol} / \mathrm{l})\end{array}$ & $\begin{array}{l}\text { Cortisol } \\
(\mathrm{nmol} / \mathrm{l})\end{array}$ & $\begin{array}{c}\text { Prolactin } \\
(u g / l)\end{array}$ & $\begin{array}{l}\text { SHBG } \\
(\mathrm{nmol} / \mathrm{l})\end{array}$ & $\begin{array}{c}\text { Epinephrine } \\
(\mathrm{pmol} / \mathrm{l})\end{array}$ & $\begin{array}{l}\text { Nor-EPI } \\
(\mathrm{nmol} / \mathrm{l})\end{array}$ & $T / S H B G$ \\
\hline \multirow[t]{2}{*}{ Rest (T-15) } & SCI & $24.1 \pm 3.5$ & $408.5 \pm 69.6$ & $7.8 \pm 1.2$ & $44.2 \pm 6.7$ & $332.4 \pm 105.1$ & $2.5 \pm 0.3$ & $0.55 \pm 0.03$ \\
\hline & $\mathrm{AB}$ & $15.3 \pm 1.7$ & $420.2 \pm 71.0$ & $10.0 \pm 1.8$ & $47.5 \pm 2.3$ & $1026.1 \pm 40.7$ & $4.9 \pm 0.5$ & $0.32 \pm 0.02$ \\
\hline \multirow[t]{2}{*}{ Pre-exercise } & SCI & $25.0 \pm 3.2$ & $373.7 \pm 73.9$ & $7.3 \pm 0.5$ & $46.7 \pm 10.5$ & $787.8 \pm 316.0$ & $2.9 \pm 0.4$ & $0.60 \pm 0.07$ \\
\hline & $\mathrm{AB}$ & $17.1 \pm 1.3$ & $437.2 \pm 59.9$ & $10.0 \pm 2.0$ & $47.5 \pm 2.9$ & $142.8 \pm 58.5$ & $4.6 \pm 0.4$ & $0.36 \pm 0.02$ \\
\hline \multirow[t]{2}{*}{ Exercise 1} & $\mathrm{SCI}$ & $27.6 \pm 3.7$ & $335.7 \pm 60.9$ & $6.8 \pm 1.2$ & $44.5 \pm 6.7$ & $546.9 \pm 31.1$ & $6.6 \pm 1.2$ & $0.64 \pm 0.08$ \\
\hline & $\mathrm{AB}$ & $18.0 \pm 1.4$ & $446.5 \pm 64.5$ & $8.7 \pm 1.7$ & $49.9 \pm 3.9$ & $1102.5 \pm 398$ & $9.0 \pm 2.5$ & $0.36 \pm 0.02$ \\
\hline \multirow[t]{2}{*}{ Rest 1} & $\mathrm{SCI}$ & $27.6 \pm 4.6$ & $353.5 \pm 73.4$ & $6.5 \pm 1.2$ & $49.3 \pm 9.1$ & $835.0 \pm 234.6$ & $2.6 \pm 0.5$ & $0.56 \pm 0.04$ \\
\hline & $\mathrm{AB}$ & $16.8 \pm 0.8$ & $413.6 \pm 48.3$ & $9.5 \pm 1.7$ & $50.0 \pm 2.9$ & $153.0 \pm 43.6$ & $5.1 \pm 0.7$ & $0.34 \pm 0.02$ \\
\hline \multirow[t]{2}{*}{ Exercise 2} & $\mathrm{SCI}$ & $30.6 \pm 5.4$ & $339.5 \pm 68.4$ & $7.3 \pm 1.2$ & $48.3 \pm 8.7$ & $992.3 \pm 382.1$ & $6.8 \pm 1.1$ & $0.63 \pm 0.02$ \\
\hline & $\mathrm{AB}$ & $18.6 \pm 1.3$ & $455.0 \pm 65.3$ & $8.6 \pm 1.2$ & $49.7 \pm 3.1$ & $862.4 \pm 365.6$ & $9.2 \pm 2.6$ & $0.38 \pm 0.30$ \\
\hline \multirow[t]{2}{*}{ Rest 2} & $\mathrm{SCI}$ & $30.6 \pm 5.3$ & $407.7 \pm 116.3$ & $7.0 \pm 1.2$ & $48.8 \pm 7.8$ & $449.7 \pm 163.7$ & $3.7 \pm 1.1$ & $0.63 \pm 0.04$ \\
\hline & $\mathrm{AB}$ & $18.2 \pm 1.9$ & $339.6 \pm 46.7$ & $7.9 \pm 0.6$ & $50.5 \pm 3.0$ & $544.7 \pm 234.7$ & $4.6 \pm 0.9$ & $0.36 \pm 0.03$ \\
\hline \multirow[t]{2}{*}{ Exercise 3} & $\mathrm{SCI}$ & $30.1 \pm 5.2$ & $383.4 \pm 96.4$ & $6.5 \pm 0.5$ & $47.1 \pm 7.4$ & $802.3 \pm 409.0$ & $8.8 \pm 1.9$ & $0.63 \pm 0.02$ \\
\hline & $\mathrm{AB}$ & $19.1 \pm 1.9$ & $334.4 \pm 66.9$ & $9.3 \pm 1.0$ & $50.5 \pm 2.3$ & $1080.0 \pm 267$ & $10.5 \pm 2.5$ & $0.37 \pm 0.05$ \\
\hline Recovery & SCI & $28.5 \pm 4.6$ & $351.2 \pm 99.1$ & $6.8 \pm 0.6$ & $52.4 \pm 10.9$ & $340.0 \pm 98.0$ & $3.6 \pm 0.7$ & $0.56 \pm 0.02$ \\
\hline$(\mathrm{T} \pm 15)$ & $\mathrm{AB}$ & $17.1 \pm 1.9$ & $261.1 \pm 50.9$ & $7.7 \pm 0.8$ & $46.9 \pm 2.2$ & $904.9 \pm 37.1$ & $6.5 \pm 2.4$ & $0.37 \pm 0.05$ \\
\hline
\end{tabular}

$8.8 \pm 1.9 \mathrm{nmol} / \mathrm{l}$ after the third exercise bout in SCI and from $4.9 \pm 0.5$ to $10.5 \pm 2.5 \mathrm{nmol} / 1$ after the third exercise bout in AB. A short half life of NOR resulted in close to baseline values following each exercise. EPI levels were unchanged with exercise in both SCI and AB.

\section{Discussion}

The present study demonstrated a significant serum testosterone response to FES assisted physical activity in wheelchair using subjects. This normal ability to respond to physical activity with increased testosterone levels presumably provides a normal hormonal stimulus of the regeneration/repair ability within the limitations of the wheelchair users' neurological disability. This should provide encouragement for both researchers and subjects involved in FES.

Questions do, however, remain. Exercise associated changes in circulating testosterone levels vary according to the nature, severity and duration of the activity and the fitness of the individual. In the present study FES-assisted resistance training induced an increase in serum testosterone levels without change in sex hormone binding globulin levels or hematocrit. While this suggests the increment is related to increased production or decreased clearance, it excludes changes in circulatory binding as responsible for the testosterone increment. SCI subjects in the present study reached or exceeded circulating norepinephrine levels associated with suppression of response in our previous investigation. ${ }^{11}$ This tends to suggest that norepinephrine alone is not a factor in suppressing the testosterone response in exercising wheelchair athletes under conditions of self-induced autonomic dysreflexia. The differences between these two clinical situations require further investigation.
Serum testosterone levels were consistently lower in the able-bodied subjects than in SCI subjects. Basal mean serum testosterone levels in SCI individuals have been described as being higher than, ${ }^{19}$ similar to,,, $20-22$ and lower $\operatorname{than}^{8-10}$ in AB subjects. In the present study both SCI and AB control subjects had mean testosterone levels in the normal range. The lower but still physiological serum testosterone levels in controls may result from the use of control subjects who were predominantly endurance trained. Basal serum testosterone values in the SCI in the present study were also higher than those previously described in elite SCI wheelchair athletes ${ }^{11}$ maintaining the difference frequently found between able-bodied, endurance trained athletes and sedentary individuals. ${ }^{23}$

The present study provides evidence that serum testosterone response to an exercise load is not impaired by exercise induced high norepinephrine levels in SCI subjects undergoing FES assisted maximal resistance exercise. The findings raise further questions about mechanisms of testosterone change with activity but do provide reassurance that serum testosterone response to activity appears normal in SCI subjects undergoing FES assisted activity.

\section{Acknowledgements}

This research was supported in part by the Recreation Parks and Wildlife Foundation, Alberta Lotteries, The Harvey Decock Society, Edmonton and the Spinal Cord Injury Treatment Centre Society (SCITCS), Edmonton. EA Ashley was supported by grants from the Faculty of Medicine and the Rick Hansen Centre, University of Alberta, Edmonton, Canada. 


\section{References}

1 Cumming DC, Wheeler GD. Exercise, training, and the male reproductive system. In: (Bouchard $\mathrm{C}$ et al. eds), Physical Activity, Fitness, and Health. Human Kinetics Publishers, Champaign, IL. 1994; pp. 980-992.

2 Cumming DC, Wheeler GD, Harber VJ. Physical activity, nutrition, and reproduction. In: Human Reproductive Ecology, Interactions of environment, fertility, and behavior (Kenneth L Campbell, James W Wood eds). Ann N Y Acad Sciences 1994; 709: $55-76$.

3 Ponjee GAE, de Rooy HAM, Vader HL. Androgen turnover during marathon running. Med Sci Sports Exerc 1994; in press.

4 Adlercreutz $\mathrm{H}$ et al. Effect of training on plasma anabolic and catabolic steroid hormones and their responses during physical exercise. Int J Sports Med 7 (suppl): 1986; 27-28.

5 Bugaresti JM et al. Changes in thyroid hormones, thyroid stimulating hormone and cortisol in acute spinal cord injury. Paraplegia 1992; 30: $401-409$.

6 Hayes PJ et al. Testicular endocrine function in paraplegic men. Clin Endocrinol 1979; 11: 549-552.

7 Finsen V, Indredavik B, Fougner KJ. Bone mineral and hormone status in paraplegics. Paraplegia 1992; 30: 343-347.

8 Naftchi NE, Viau AT, Sell GH, Lowman EW. Pituitary-testicular axis dysfunction in spinal cord injury. Arch Phys Med Rehabil 1980; 61: 402-405.

9 Naftchi NE. Alterations of neuroendocrine functions in spinal cord injury. Peptides 1985; 6: (Suppl 1) pp. 85-94.

10 Claus-Walker J, Scurry M, Carter RE, Campos RJ. Steady state hormonal secretion in traumatic quadriplegia. J Clin Endocrinol Metab 1977; 44: 530 - 535.

11 Wheeler $\mathrm{G}$ et al. Testosterone, cortisol and catecholamine responses to exercise stress and autonomic dysreflexia in elite quadriplegic athletes. Paraplegia 1994; 32: 292-299.

12 Twist DJ et al. Neuroendocrine changes during functional electrical stimulation. Am J Phys Med Rehab 1992; 71: 156-163.
13 Mathias CJ et al. Plasma catecholamines during paroxysmal neurogenic hypertension in quadriplegic man. Circul Res 1976; 39: $204-208$.

14 Phillips CA, Petrofsky JS, Hendershot DM, Stafford D. Functional electrical exercise - a comprehensive approach for physical conditioning of the spinal cord injured patient. Orthopaedics 1984; 7: 1112-1123.

15 Petrofsky JS, Brown SW, Cerel-Bazo H. Active physical therapy and its benefits in rehabilitations. Palaestra 1992; 8: 23-32.

16 Ragnarsson KT et al. Clinical evaluation of computerized electrical stimulation after spinal cord injury: a multicenter pilot study. Arch Phys Med Rehabil 1988; 69: 672-677.

17 Ashley EA et al. Evidence of autonomic dysreflexia during functional electrical stimulation in individuals with spinal cord injuries. Paraplegia 1993; 31: 593-605.

$18 \mathrm{McColl}$ EM et al. The effects of acute exercise on LH pulsatile release in high mileage male runners. Clin Endocrinol 1989; 31: 617-629.

19 Perkash I et al. Reproductive biology of paraplegics: results of semen collection, testicular biopsy and serum hormone evaluation. J Urol Aug 1985; 134 (2): pp. 284-288.

20 Nance PW, Shears AH, Givner ML, Nance DM. Gonadal regulation in men with flaccid paraplegia. Arch Phys Med Rehabil 1985; 66 (11): pp. 757-759.

21 Young RJ et al. Is testicular endocrine function abnormal in young men with spinal cord injuries? Clin Endocrinol (Oxf) Sep 1982; 17 (3): pp. $303-306$.

22 Chappelle PA et al. Trophic effects on testes in paraplegics. Paraplegia 1993; 31: 576- 583.

23 Wheeler GD, Wall SR, Belcastro AN, Cumming DC. Reduced serum testosterone and prolactin levels in male distance runners. JAMA 1984; 252: 514-516. 